\title{
Low Frequency Oscillation Modal Parameter Identification Based on NExT-ERA and Fuzzy Clustering
}

\author{
Gao Jie ${ }^{1}$, Wang $\mathrm{Jia}^{2}$ and Zhou Yang ${ }^{1}$ \\ ${ }^{1}$ School of Electrical Engineering, Southwest Jiaotong University, Chengdu \\ 610031, China \\ ${ }^{2}$ Sichuan Electric Power Company \& Measuring Center, Chengdu 610045, China \\ jiegaow@yeah.net
}

\begin{abstract}
Using ambient excited data under PMU measurements to identify the low frequency oscillation mode and oscillation modes parameter information corresponding, has good prospects in power system analysis and control. This article discusses the applicability by using the natural excitation technique (NExT) in conjunction with the eigensystem realization algorithm for low frequency oscillation modes identification, then introduced fuzzy $C$-means clustering algorithm to picked up the authenticity of the identified modal results automatically and improving the recognition accuracy. On the IEEE-11 and IEEE-68 bus test system numerical example shows that the proposed method has higher modal recognition ability and efficiency, and can meet the needs of online applications.
\end{abstract}

Keywords: low frequency oscillation; modal analysis; ambient excited; Natural Excitation Technique; Eigensystem Realization Algorithm; fuzzy clustering.

\section{Introduction}

With the interconnection of the power grid region and the weakening of the damp, the instable increasing oscillation has been a frequent occurrence of the system. Therefore, it is of vital importance and more difficult for the online monitoring of the power grid and the damping control to rapidly obtains the low frequency oscillation models and parameters [1].

With the all-around application of Phasor Measurement Unit (PMU) in the electrical power system and the gradual establishment of Wide Area Monitoring Systems (WAMS) based on PMU, the analysis of the system's low frequency oscillation based on the actually-measured tracks enjoys a promising prospect. According to different disturbance intensity, there are two categories of identification methods, namely the identification method based on the large signal disturbance signal and the identification method based on the small disturbance signal [2]. Currently, Prony has been the widely-used one among the low frequency oscillation modal identification methods based on the large oscillation disturbance. However, since the large disturbance signal might not exit all the time and the actually-measured signal is seriously impacted by the environment noises, the limits of Prony and the defects of data collected by it have been increasingly obvious. The modal identification methods based on the noise-like signal only adopt the real-time response signal in ambient excitation as the recognition input, and have no need to measure the input the drive signal. At the same time, it can avoid the complexity of manual excitation. Thus, methods of the kind are more applicable to the online monitoring and analysis of the low frequency oscillation model [3].

Based on the above findings, experts and scholars have made some explorations in the relevant fields. Literature [4] introduce Auto Regressive Moving Average (ARMA) to describe the random signal, and use the least square method to estimate 
the parameters, but the identification accuracy has a poor robustness due to the huge impact of noises. Literature [5] replaces the frequency response function with the auto-cross power spectrum between the output of the measurement points to identity modal parameters, but there are defects, such as huge damping recognition error and low computation efficiency. Literature [6] introduces the random subspace method into the electrical power system. The method has certain anti-jamming capacity against output noises, and boasts unique advantages in terms of identifying modal information. However, in the practical system, the input noise excitation cannot strictly meet the requirement of the zero-mean. Thus, the false identification modal can be easily generated. Methods put forward in Literature [4] and [5] both conduct modal identification based on the measurement of the single signal and the system's dynamic linear model, but, to achieve the optimal identification, the single signal measurement is inadequate. Literature [6] and [7] put forth the method of increasing the measurement channels and employing multiple variables to improve the identification effect of the system modal, but the complex computation is still a problem.

This paper puts forward a NExT-ERA low frequency oscillation noise-like identification method based on the fuzzy clustering. The method obtains the system's impulse response function through the NExT of the reference channel signals. Then, ERA identification is conducted of the system under different orders. At last, FCM is adopted to conduct the clustering analysis of the identification results to automatically collect he authentic modal. First, the feasibility of the method is expounded theoretically; second, the method's validity is verified based on the analysis of the data gathered by the synchronic and asynchronous measurement. Besides, the method is proved to have a good noise resistant effect.

\section{FCM-based NExT-ERA Modal Identification}

\subsection{Natural Excitation Technique (NExT)}

Natural Excitation Technique or NExT [8] has been found successful applications in the civil engineering and machinery field $[9,10]$, but it has not yet been widely used to recognize the modal parameters of the electrical power. After the electrical power system suffers little disturbance, the swaying equation of its generator set's rotor is similar to the dynamic differential equation of the linear time-invariant vibration system with the degree of freedom of "n." Besides, the generation of the low frequency oscillation is similar to that of general vibration mechanically. Therefore, to analyze the low frequency oscillation of the electrical power system can refer to the research findings of the modal analysis in the field of dynamics. In the following part, the feasibility of NExT in the modal identification of the low frequency oscillation will be first studied.

To analyze the low frequency oscillation of the electrical power system, the following differential equation can be used to describe the oscillation motor equation of the single generator set's rotor:

$$
M \frac{d^{2} \delta}{d t^{2}}=P_{M}-P_{E}-D \frac{d \delta}{d t}
$$

Where, $\mathrm{M}$ stands for the inertia constant; PM for the turbine power; PE for the air gap power; D for the damping coefficient; and $\delta$ for the rotor angle. The typical constant magneto model is adopted to conduct linearization around the operating points. The following equation can be obtained:

$$
M \frac{d^{2} \Delta \delta}{d t^{2}}+D \frac{d \Delta \delta}{d t}+K \Delta \delta=0
$$


Where, K stands for the constant of the synchronizing power, and for the rotor's angular displacement of the equilibrium point.

The actual motion state of the electric power system is not in a full steady state. At any time, every generator set is subject to randomly-growing power excitation, namely ambient environment. The random variation of the load with the characteristic similar to that of the white noise is a major excitation. Besides, the subtranient will also be regarded as the system's natural excitation, such as the subtle changes of the operation model, some switch events and some faults. Considering the natural excitation, the angular oscillation equation of the rotor with the degree of freedom of $n$ will be expanded by Eq. (2) into the following one:

$$
M \Delta \delta(t)+D \Delta \delta(t)+K \Delta \delta(t)=F(t)
$$

Where, $F(t)$ is the exiting force vector; M, D and $\mathrm{K}$ stand for the inertial matrix, damping matrix and synchronizing power matrix of the generator set, respectively, which are all " $n \times n$ " order symmetric matrix; $(\cdot)$ stands for the derivation of time; $\Delta \delta(t), \Delta \delta(t)$ and $\Delta \delta(t)$ stand for the " $\mathrm{n}$ " order displacement, speed and acceleration array of the rotor angle. Multiple Eq.(3) with a referential rotor's angular displace, $\Delta \delta_{r}(s)$, on the right. Get the expected value of every part to obtain the following equation:

$$
M E\left[\Delta \delta(t) \Delta \delta_{r}(s)\right]+D E\left[\Delta \delta(t) \Delta \delta_{r}(s)\right]+K E\left[\Delta \delta(t) \Delta \delta_{r}(s)\right]=E\left[F(t) \Delta \delta_{r}(s)\right]
$$

Where, $E[\square]$ stands for the mathematical value of expectation. Eq. (4) can also be expressed as follows:

$$
M R_{\Delta \delta \Delta \delta_{r}}(t, s)+D R_{\Delta \delta \Delta \delta_{\delta_{r}}}(t, s)+K R_{\Delta \Delta \Delta \delta_{r}}(t, s)=R_{F \Delta \delta_{r}}(t, s)
$$

Where, $R(\square)$ stands for the vector of the cross-correlation function.

Suppose $A(t)$ and $B(t)$ are a stationary process, then

$$
R_{A^{(m)} B}(\tau)=R_{A B}^{(m)}(\tau)
$$

Where, $\tau=t-s, \quad A^{(m)}$ stands for the " $m$ " $\left(m=1^{s t}, 2^{\text {nd }}, 3^{\text {rd }} \ldots\right)$ derivation of time by $A(t)$ during the random process; and $R_{A B}^{(m)}$ stands for the " $m$ " $\left(m=1^{s t}, 2^{\text {nd }}, 3^{\text {rd }} \ldots\right)$ derivation of $\tau$ by the cross-correlation function, $R_{A B}(\tau)$.

When $\tau>0$, and the natural excitation of the system is the white noise, the referential angular displacement, $\Delta \delta_{r}(s)$, is irrelevant to $F(t)$. In other words, when $\tau>0, R_{F \Delta \delta_{r}}(t, s)=0$. Besides, assuming that the rotor's angular displacement is $\Delta \delta(t)$; speed,$\Delta \delta(t)$; and acceleration; $\Delta \delta(t)$. All are in a stationary process, then Eq. (5) is:

$$
M R_{\Delta \delta \Delta \delta_{r}}(\tau)+D R_{\Delta \delta \Delta \delta_{r}}(\tau)+K R_{\Delta \delta \Delta \delta_{r}}(\tau)=0, \tau>0
$$

Therefore, the cross-correlation function of different rotor's angular displacement, $R_{\Delta \delta \Delta \delta_{r}}(\tau)$, is similar to the oscillation differential equation of the generator set's rotor, and $R_{\Delta \delta \Delta \delta_{r}}(\tau)$ can replace the impulse response function to be used for the identification of the time domain modal parameters. Around the operation points, the angle of the generator set's rotor and the other physical 
variables of the system will generate linear coupling effect, such as power tide, voltage, etc. Therefore, the cross-correlation function of the output response of these physical variables can replace their impulse response function to conduct modal identification of the system.

NExT should choose the reference variables to obtain the cross-correlation function, such as the referential angular displacement, $\Delta \delta_{r}$, in Eq. (7). When the system suffers natural excitation, the referential variables measured by PMU have certain modal observation value and a high signal-to-noise ratio.

\subsection{Eigen Realization Algorithm (ERA)}

Eigen Realization Algorithms (ERA) [11] were put forward by Juang et al., of the Langley Research Center in the US in 1984. In essence, it employs the multiple-inputmultiple-output (MIMO) impulse response function to build the Hankle matrix, and the singular value decomposition (SVD) to decompose and seek a minimal realization of the system, (A, B, C) [12]. Based on that, the eigenvalue problem of the system's matrix, A, can be solved.

The state space model of the system's discrete time is

$$
\begin{gathered}
x(k+1)=A x(k)+B u(k) \\
y(k)=C x(k)
\end{gathered}
$$

$x(k)$ stands for the system's time state vector at the moment of $k \Delta t ; u(k)$ stands for the system's excitation vector at the moment of $k \Delta t ; y(k)$ stands for the system's state output vector at the moment of $k \Delta t ; \mathrm{A}, \mathrm{B}$ and $\mathrm{C}$ are the state matrixes of the system.

Use the pulse response matrix with the order of $h(k)$ and $m \times r$, respectively, to form the Hankle matrix:

$$
H(k-1)=\left[\begin{array}{cccc}
h(k) & h(k+1) & \cdots & h(k+\beta-1) \\
h(k+1) & h(k+2) & \cdots & k(k+\beta) \\
h(k+2) & h(k+3) & \cdots & h(k+\beta+1) \\
\vdots & \vdots & & \vdots \\
h(k+\alpha-1) & h(k+\alpha) & \cdots & h(k+\alpha+\beta-2)
\end{array}\right]
$$

The order of the matrix, $H(k-1)$, is $\alpha m \times \beta r ; \alpha$ and $\beta$ stand for the observable index and the controllable index, respectively; and $2 n / m \leq \alpha \leq 2 n, 2 n / r \leq \beta \leq 2 n$.

When $k=1$, the SVD of the data matrix leads to the following results:

$$
H(0)=P \sum Q^{\mathrm{T}}
$$

Where, $P$ and $Q$ stand for the orthonormal matrix; and $\Sigma$ is the rectangular matrix.

$$
\Sigma=\left[\begin{array}{cc}
\Sigma_{n} & 0 \\
0 & 0
\end{array}\right]
$$

$\Sigma_{n}=\operatorname{diag}\left(\sigma_{1}, \sigma_{2}, \cdots, \sigma_{i}, \sigma_{i+1}, \cdots, \sigma_{n}\right), \sigma_{i}$ is the singular value of $H(0)$. Choose the maximum integer, $2 n$, as the system's order. Pn and Qn constitute of the former $n$ rows of the matrix, $P$ and $Q$. Therefore, the matrix, $H(0)$, and its generalized inverse, $H^{+}$, can be written as below:

$$
\begin{gathered}
H(0)=P_{n} \sum_{n} Q_{n}^{\mathrm{T}}, \quad P_{n}^{\mathrm{T}} P_{n}=I_{n}=Q_{n}^{\mathrm{T}} Q_{n} \\
H^{+}=P_{n} \Sigma_{n}^{-1} Q_{n}^{\mathrm{T}}
\end{gathered}
$$


Observe Eq. (13) and Eq. (14). When k=1,

$$
H(1)=P_{\alpha} A Q_{\beta}=P_{n} \sum_{n}^{1 / 2} A \sum_{n}^{1 / 2} Q_{n}^{\mathrm{T}}
$$

The system's minimal realization can be obtained:

$$
\left\{\begin{array}{c}
A_{1}=\sum_{n}^{-1 / 2} P_{n}^{\mathrm{T}} H(1) Q_{n} \Sigma_{n}^{-1 / 2} \\
B_{1}=\Sigma_{n}^{-1 / 2} Q_{n}^{\mathrm{T}}\left[\begin{array}{ll}
I & 0
\end{array}\right]^{\mathrm{T}} \\
C=\left[\begin{array}{ll}
I & 0
\end{array}\right] P_{n} \sum_{n}^{-1 / 2}
\end{array}\right.
$$

Assume the eigenvalue matrix of the matrix, $A_{1}$, is $Z$, and the eigenvector matrix is ${ }^{\psi}$, both of which are of the order of $2 n \times 2 n$, then

$$
\psi^{-1} A_{1} \psi=\psi^{-1} e^{A_{1} \Delta t} \psi=Z
$$

The eigenvalue of the system matrix, $A_{1}$, is $\lambda_{\mathrm{i}}$ :

$$
\lambda_{i}=\ln \left(\frac{Z}{\Delta t}\right)=\lambda_{i}^{\mathrm{Re}}+j \lambda_{i}^{\mathrm{Im}}
$$

Then, the undamped inherent frequency and the damping ratio of different modals are shown below:

$$
\begin{gathered}
\omega_{i}=\frac{1}{2 \pi} \sqrt{\left(\lambda_{i}^{\mathrm{Re}}\right)^{2}+\left(\lambda_{i}^{\mathrm{Im}}\right)^{2}} \\
\xi_{i}=\frac{\lambda_{i}^{\mathrm{Re}}}{\omega_{i}}
\end{gathered}
$$

\subsection{Selection of the Reference Channel}

ERA is a MIMO time-domain overall modal parameter identification algorithm. Its input is the cross-correlation function matrix build by the reference channel vectors (multiple reference points), which can thus improve the accuracy and reliability of the identification results.

Under the environment excitation, the input drive signal of the electrical power system is similar to the white noise, and cannot be measured. In order to obtain the modal of a certain system, it is necessary to include all the signals measured by the electric generator's PMUs into the system's output column vectors. However, in fact, there is huge number of PMUs in the electric power system. It is extremely time-consuming to include the measured signals as the signals of the reference channels into $h(\mathrm{k})(m \times 1)$ to form a Hankel matrix and conduct SVD. This might also hinders its online application. Therefore, it is an issue of great concern about how to quickly select signals with a high observability and representativeness from the mass data measured by PMUs as the signals of the reference channels.

The selection of the reference channels is based on a preliminary understanding of the system. First, derive the estimation methods of the dominant oscillation models based on the system's mathematical models; find the key fracture surfaces which might easily oscillate with the system through the analysis of the factors influencing the oscillation frequency; select the measured tracks on the fracture surface based on the oscillation increase amount of the state quantity [13]. After all these, the signals for the reference channels with a high observability towards the oscillation model can be confirmed.

\subsection{Identification Accuracy Indexes of the Modal Parameters}

Since the actually-measured signals might inevitably be influenced by the environment noises and the system's nonlinear factors, there might be difficulties with the order 
determination while conducting SVD of $\mathrm{H}(0)$. If the singular value decreases suddenly at certain order, the mutation is the system's order; if there is no obvious mutation, the order cannot be directly decided. Therefore, Juang et al., [14] introduced Modal Amplitude Coherence (MAC), Extended Modal Amplitude Coefficient (EMAC) and Modal Phase Colinearity (MPC) and Consistent Modal Index (CMI) [15] to distinguish the real modal and the noise modal among the identification results.

This paper employs NExT-ERA to identity modal parameters at different orders. The modal parameters with a high credibility is preliminarily selected out according to the threshold value, " $0 \%<$ damping ratio< $10 \%$ \& CMI $>70 \%$," and enter the next step of judgment.

\subsection{Selection of Physical Modals based on the FCM Algorithm}

The traditional authentic and false modal identification method is to draw every group of modal parameters recognized at different orders on the stability diagram, and manually choose the stable points from the diagram, the corresponding modal of which is the system's authentic modal. However, rich practical experiences are called for the treatment of the stability diagram. Selection of improper stable points will influence the accuracy of the identification results, which will be unfavorable for the automatic pickup of the modal parameters. In response to the problem, this paper introduces FCM into the identification of authentic and false modals, adopt modal frequencies and damping ratios identified at different orders as samples and classify them. Choose the classification center as the final identification results of the system's modal parameters. The basic principle for the authentic and false modal identification is that the identification results of the system at different orders contain the authentic modal information, and that the similar modal values can be easily gathered into a specific category. Then, choose the corresponding modal of the central point of the kind as the system's most authentic physical modal.

Fuzzy clustering has a wide application in the intelligent classification of the statistical pattern identification, of which FCM is the one with the most mature application and theoretical system. Its principle is as below [16]: define an objective function, $J$; randomly select " $c$ " initial cluster centers, $v_{i}(i=1,2, \cdots, c)$, from the sample set to be classified, $X=\left\{x_{1}, x_{2}, \cdots, x_{n}\right\} \subset R^{p}$; divide the samples to the category through the calculation of the Euclidean distance, $d_{i j}$, from the calculation samples to the cluster centers; and update and calculate cluster centers of every category at last. The iteration is repeated until the objective function can reach the minimum. The objective function, $J$, is defined as below.

$$
J\left(U, v_{1}, v_{2}, \cdots, v_{c}, X\right)=\sum_{i=1}^{c} \sum_{j=1}^{n} \mu_{i j}^{m} d_{i j}^{2}
$$

It also meets the following limiting conditions:

$$
\sum_{i=1}^{c} \mu_{i j}=1, \forall j=1,2, \cdots, n(1 \leq j \leq n)
$$

Where, $\mu_{i j} \in[0,1]$ stands for the membership function of the " $j$ " $\left(j=1^{s t}, 2^{\text {nd }}, 3^{\text {rd }} \ldots\right)$ category of samples towards the " $i$ " $\left(i=1^{s t}, 2^{\text {nd }}, 3^{\text {rd }} \ldots\right)$ category, which constitutes the membership matrix, $U ; m>1$ stands for the fuzzy parameter, which decides the fuzziness of the cluster. Under most conditions, $m=2$. Under the restriction of Eq. (22), Eq. (21) is optimized into: 


$$
\begin{gathered}
v_{i}=\frac{\sum_{j=1}^{n} \mu_{i j}^{2} x_{j}}{\sum_{j=1}^{n} \mu_{i j}^{2}} \\
\mu_{i j}=\frac{1}{\sum_{k=1}^{c}\left(\frac{d_{i j}}{d_{k j}}\right)^{\frac{2}{m-1}}}
\end{gathered}
$$

The basic steps of FCM algorithm are shown below:

Initialization of the membership matrix, $U$ : choose the number of the cluster categories, $c$; set the iteration stop valve, $\varepsilon$, and the maximum iteration times, $T$; and make the current iteration times, $t=1$.

Update the cluster center based on Eq. (23), and the following equation can be obtained:

$$
v_{i}^{(t)}=\frac{\sum_{j=1}^{n}\left(\mu_{i j}^{(t-1)}\right)^{2} x_{j}}{\sum_{j=1}^{n}\left(\mu_{i j}^{(t-1)}\right)^{2}}
$$

Calculate the distance, $d_{i j}$, namely:

$$
d_{i j}^{2}=\left\|x_{j}-v_{i}\right\|^{2}=\left(x_{j}-v_{i}^{(t)}\right)^{\mathrm{T}}\left(x_{j}-v_{i}^{(t)}\right)
$$

Update the membership matrix based on Eq. (26):

$$
\mu_{i j}^{\left({ }^{(t)}\right.}=\frac{1}{\sum_{k=1}^{c}\left(\frac{d_{i j}}{d_{k j}}\right)^{\frac{2}{m-1}}}
$$

Preset the threshold value, $\varepsilon$. If $\left\|U^{(t+1)}-U^{(t)}\right\| \leq \varepsilon$, or the iteration times reach the maximum, the iteration will be stopped; otherwise, it will return to $E q$. (25) for a new round of calculation.

Thus, it can be seen that the algorithm can realize the automatic identification of the system's modal parameters, and has a high calculation efficiency. Besides, the accuracy of the modal identification results is improved through the rectification of the authentic modal selection during the repetitive iteration process. The algorithm meets the requirements of online applications.

\section{Auto Pickup of Modal Parameters}

After the introduction of the algorithm basis and the analysis of the key techniques, this part will present the specific implementation procedures of the algorithm. (See Fig. 1)

The automatic identification steps of the algorithm include the confirmation of the reference channels, the selection of the initial reference points, the pretreatment of the delayed signal cut-off, the modal identification of NExT-ERA of the system at different orders, the initial selection of CMI and other similar indexes and the FCM analysis of the screened results. At last, the final identification results of the system's modal parameters can be obtained. 


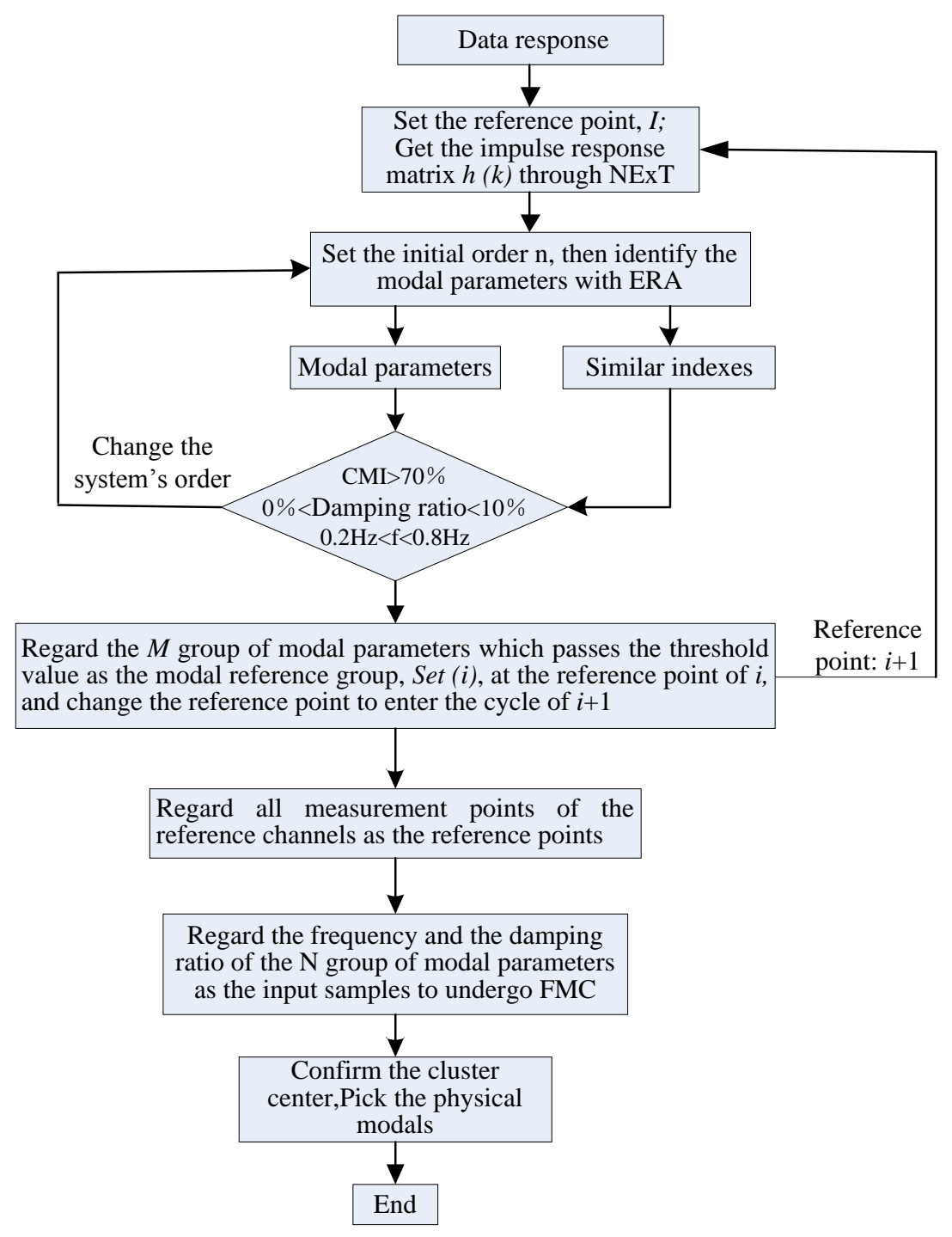

Figure 1. The Flowchart of Automatic Identification Algorithm

\section{Example Analysis}

\subsection{System with Four Generators and Four Areas}

Through the simulated data analysis of the two-area system of the IEEE 4 machine, the validity of FCM-based NExT-ERA in identifying modals put forward in this paper is verified. The system wiring diagram is shown in Fig. 2. Refer Literature [17] for specific parameters. Since the main concern of this paperis the low frequency oscillation between system areas, the eigenvalue calculation of the system's mathematical model finds that the system has a section oscillation model whose frequency is $0.6357 \mathrm{~Hz}$ and damping is 0.0131 .

Build the simulation system based on the MATLAB platform. Impose a white noise excitation at the position of the system loading. Use PMUs to measure the response data of the angular velocity of various generator sets' rotor. The sampling frequency is $100 \mathrm{~Hz}$ and the sampling time is $10 \mathrm{~min}$. Fig. 3 shows the synchronous and noise-free signals and power spectrum of the angular speed of the generator set G1's rotor. It can be seen that around $0.6 \mathrm{~Hz}$ the system has an oscillation frequency. 


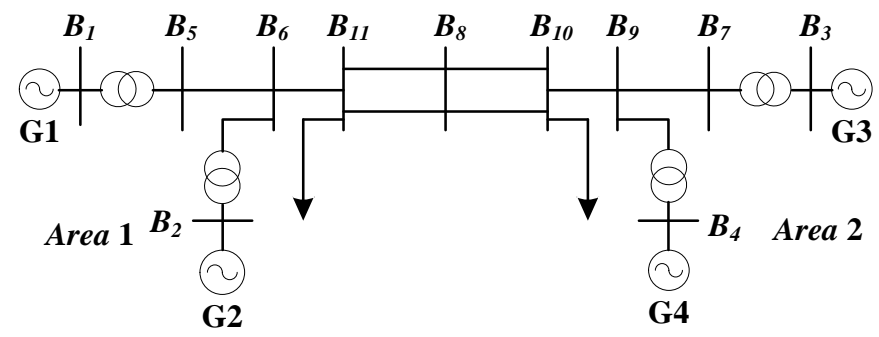

Figure 2. IEEE Four-machine Two-area Test System
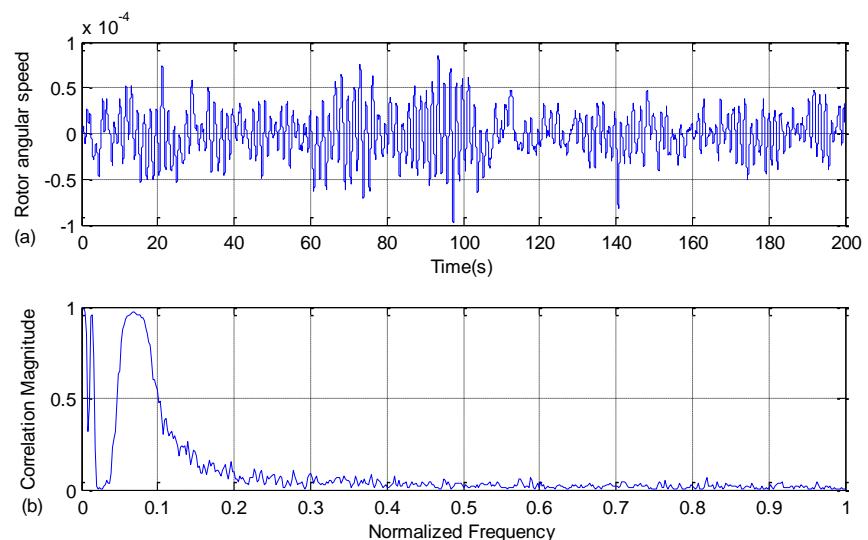

Figure 3. (a)Rotor Angle Measurement of G1and (b) The Power Spectrum Density (PSD) of the Measurement

Since the system model only has four generators, angular velocity response of the four generators' rotor is introduced into the reference channel. In order to analyze the algorithm's robustness, add $10 \mathrm{~dB}, 5 \mathrm{~dB}$ and infinitely small white Gaussian noise to every measurement channel. Then NExt-ERA modal identification is conducted of measured data whose time windown is $10 \mathrm{~min}, 5 \mathrm{~min}$ and $2.5 \mathrm{~min}$, respectively. Set the order of $\Sigma_{n}$ in Eq. (13) in 2.2 to increase progressively from 2 to 20 through ten calculations, and obtain the system's modal parameters at different orders. Set the threshold value to meet requirements of " $0 \%<$ damping ratio $<10 \% \& \mathrm{CMI}>70 \% \& 0.1 \mathrm{~Hz}<\mathrm{f}<0.8 \mathrm{~Hz}$." Select the frequency and the damping ratio of the modal parameter swhich pass the threshold as the samples and conduct FCM of them. At last, the final identification results can be obtained according to the cluster center.

By observing the cluster identification Fig. 4, it can be found that: when the data's window time is $10 \mathrm{~min}$ and $5 \mathrm{~min}$ respectively, the modal parameter samples identified through the NExt-ERA algorithm are more concentrated than those identified when the window time is $2.5 \mathrm{~min}$, and the cluster effect of the former is also more obvious. When Gaussian white noises of different $\mathrm{dBs}$ are added, the concentration of the modal samples undergoes no dramatic changes. The major reason is that, when NExT is calculating the cross-correlation function, it eliminates partial noises among signals according to the cross-correlation of the similar noise serial to be zero. During the ERA process, SVD equals to a Winner filtering, which makes the noise resistant effect of the NExT-ERA algorithm more obvious.

From the analysis results of Table 1, when the time window is $10 \mathrm{~min}, 5 \mathrm{~min}$ and $2.5 \mathrm{~min}$, respectively, the average identification error of modal parameters under different noises is $1.42 \%, 2.32 \%$ and $4.19 \%$, respectively. At the same time, the average identification error of the modal frequency is small than that of the modal damping rate. 

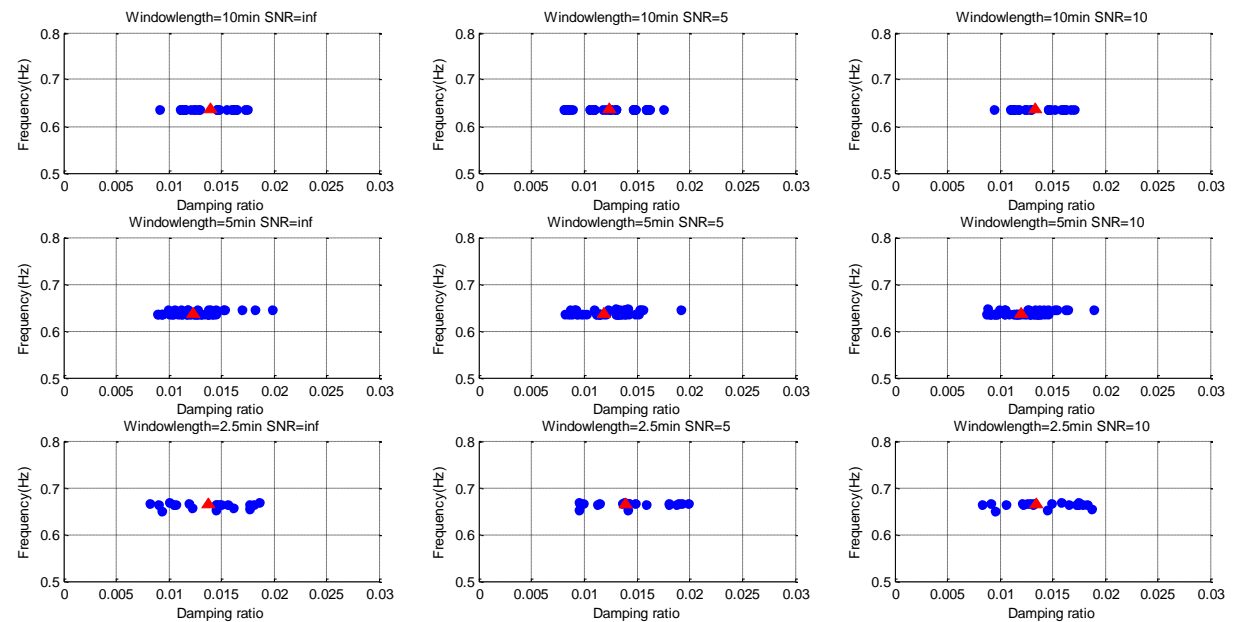

Figure 4. The Modal Analysis for the IEEE Four-machine Two-area Test System based on Clustering

Table 1. Synchronous Identification Results and Error Analysis

\begin{tabular}{|c|c|c|c|c|c|c|c|c|c|c|c|c|}
\hline \multirow{2}{*}{ SNR(dB) } & \multicolumn{5}{|c|}{ Frequency (Hz) } & \multicolumn{6}{c|}{ Damping ratio (\%) } \\
\cline { 2 - 13 } & $10 \mathrm{~min}$ & $\begin{array}{c}\text { Error } \\
(\%)\end{array}$ & 5 min & $\begin{array}{c}\text { Error } \\
(\%)\end{array}$ & $2.5 \mathrm{~min}$ & $\begin{array}{c}\text { Error } \\
(\%)\end{array}$ & $10 \mathrm{~min}$ & $\begin{array}{c}\text { Error } \\
(\%)\end{array}$ & $5 \mathrm{~min}$ & $\begin{array}{c}\text { Error } \\
(\%)\end{array}$ & $2.5 \mathrm{~min}$ & $\begin{array}{c}\text { Error } \\
(\%)\end{array}$ \\
\hline Inf & 0.6358 & 0.016 & 0.6354 & 0.047 & 0.6562 & 3.22 & 0.0129 & 1.53 & 0.0128 & 2.29 & 0.0133 & 1.53 \\
\hline 10 & 0.6358 & 0.016 & 0.6354 & 0.047 & 0.6654 & 4.67 & 0.0129 & 1.53 & 0.0124 & 5.34 & 0.0124 & 5.34 \\
\hline 5 & 0.6362 & 0.079 & 0.6362 & 0.079 & 0.6677 & 5.02 & 0.0124 & 5.34 & 0.0123 & 6.10 & 0.0138 & 5.34 \\
\hline
\end{tabular}

From the above analysis, it can be seen that the FCM-based NExT-ERA algorithm is effective in identifying modals of data synchronously measured, and that its noise resistant effect is salient. Besides, the longer the data's time window is, the more obvious the identification effect is. Compared with the identification effect of the modal's damping ratio, the identification effect of the algorithm of the modal's frequency is better. At the same time, only $0.635 \mathrm{~Hz}$ of modal frequency exists in the identification results. This suggests that, under the environment excitation, the system with four generators and two areas has a section frequency of $0.6357 \mathrm{~Hz}$, which is the major modal for the system's prevailing research.

\subsection{System with 16 Generators and 68 Nodes}

The IEEE New England-New York System featuring 16 generators and 68 nodes is shown in Fig. 5.[18] The system has 33 loads in total, of which seven are set as random ones, whose active power output accounts for about $67.6 \%$ of the total. Build the simulation system based on the MATLAB platform, and add the Gaussian white noise to seven random loads to obtain the output response of the angular velocity of the rotor of various generator sets. The data sampling frequency is $100 \mathrm{~Hz}$, and the time window length is $10 \mathrm{~min}$. Similarly, through the eigenvalue analysis of the system's mathematical model, the frequency and the damping ration of the low frequency oscillation dominant models of the system are obtained. (See Table 2) 


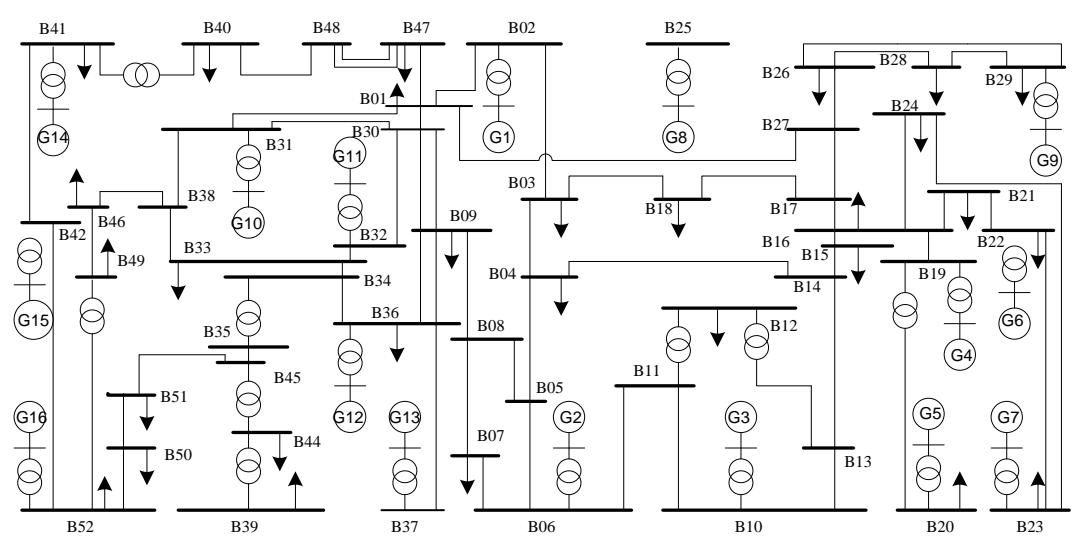

Figure 5. IEEE 16 Machine 68 Nodes Test System

Table 2. Dominant Modes for Low-frequency Oscillation

\begin{tabular}{|c|c|c|c|}
\hline Model & Frequency $(\mathrm{Hz})$ & Damping ratio(\%) & Participating generator set \\
\hline 1 & 0.3881 & 0.0502 & G1,G4,G15 \\
\hline 2 & 0.5217 & 0.0085 & G5,G13,G16 \\
\hline 3 & 0.6768 & 0.0391 & G1-G9,G12,G13 \\
\hline 4 & 0.7930 & 0.0353 & G14,G15,G16 \\
\hline
\end{tabular}

Since the increase of the number of the system's generator sets, if the cross-correlation function between the reference points and the angular velocity response of the rotor of all the other generator sets is first solved before ERA calculation is conducted, the calculation amount will be huge. This is also not beneficial for the identification of the dominant model. Based on the preliminary understanding of the system structure (See Table 2), it is found that the system's dominant models exist in some generator sets. Therefore, this paper adopts measurement signals of the rotor's angular velocity of ten generator sets (No. 1, 4, 5, 6, 11, 13, 14, 15 and 16) with the maximum observability among the area oscillation models as the signals of the reference channels. Then, the algorithm put forward in this paper is adopted to conduct modal identification.

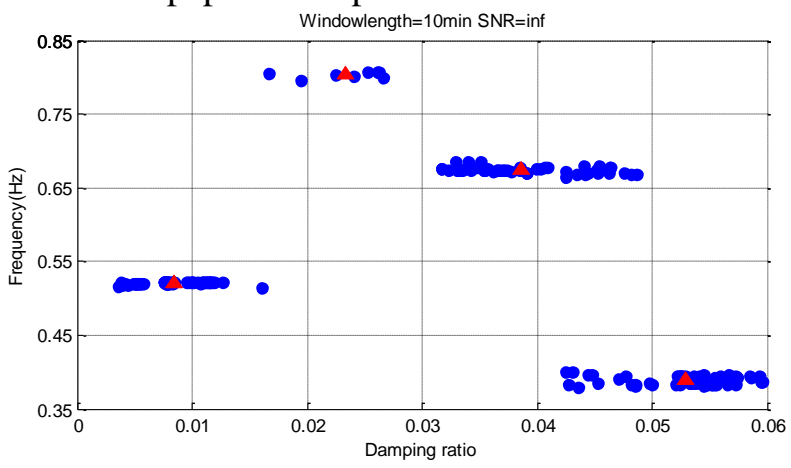

Figure 6. Clustering of Modal Identification

Table 3. Clustering Results of the Modal Identification and Error Analysis

\begin{tabular}{|c|c|c|c|c|}
\hline Modal & Frequency $(\mathrm{Hz})$ & Error $(\%)$ & Damping ratio(\%) & Error (\%) \\
\hline 1 & 0.3837 & 1.13 & 0.0525 & 4.58 \\
\hline 2 & 0.5215 & 0.04 & 0.0084 & 1.18 \\
\hline 3 & 0.6747 & 0.31 & 0.0386 & 1.28 \\
\hline 4 & 0.8021 & 1.45 & 0.0240 & 31.8 \\
\hline
\end{tabular}


By observing the cluster identification Fig. 6, it can be found that: the number of modular parameter samples identified of Modal 1, Modal 2 and Modal 3 through the NExT-ERA algorithm is larger; moreover, the modal frequency samples are relatively concentrated while the damping ratio samples are relatively diverging. The analysis results of Table 3 show that the algorithm has a high overall identification accuracy of modal frequency, and that its identification error of the damping ratio of Modal 4 is far larger than that of the other three modals.

From the above analysis, it can be learned that the FCM-based NExT-ERA algorithm is also applicable to the identification of multiple oscillation modals. When the system scale expands, the signals of the reference channels with a higher observability are adopted for modal identification, which can not only improve the calculation speed, but also realize high identification accuracy.

\section{Conclusions}

This paper puts forward the FCM-based NExT-ERA algorithm to conduct modal identification of low frequency oscillation noises. Under the environment excitation, the signals with a higher observability are selected from the data measured by PMUs as the signals of the reference channels. NExT is employed to obtain the cross-correlation function between signals so as to obtain the approximate pulse response function of the system. Then, ERA is adopted to conduct modal parameter identification of the pulse response at different orders. At last, the FCM algorithm is introduced to conduct automatic pickup of all identification results, which can not only identify the authenticity of modals, but also improve the parameter identification accuracy. The validity of the algorithm is verified through the simulation examples.

Besides, the algorithm put forward in this paper has advantages in the following four aspects:

1) The algorithm adopts the random loads generated by the electric power system as the natural excitation to avoid the complexity of the manual excitation. Besides, the parameters identified by it are more suitable for the operation conditions. Due to the limitation of algorithms, the modal identification in the previous literatures is mostly targeted at measurement signals based on large disturbance.

2) The algorithm has a sound noise resistant performance. When the Gaussian white noise is added into the signal, with the decrease of the signal-noise ratio (SNR) added, the algorithm can more accurately identify the modal parameters.

3) During the identification process of the authentic and false models, this paper introduces the FCM-based automatic identification algorithm, which improves the identification accuracy and the calculation efficiency. After setting some initial parameters, the algorithm put forward by this paper can achieve full automation and call for no manual intervention, so it boasts a promising online application prospect.

\section{References}

[1] F. Zhu, H. G. Zhao and Z. H. Liu, "The Influence of Large Power Grid Interconnected on Power System Dynamic Stability", Proceedings of the CSEE, (2007).

[2] J. M. Ni, C. Shen and F. Liu, "Estimation of the electromechanical characteristics of power systems based on a revised stochastic subspace methodand the stabilization diagram", Sci China Tech. Sci., vol. 55, (2012), pp. 1677-1687.

[3] J. W. Pierre, D. J. Trudnowski and M. K. Donnelly, "Initial results in electromechanical mode identification from ambient data", IEEE Trans Power Syst., vol. 12, no. 3, (1997), pp. 1245-1251.

[4] R. W. Wies, J. W. Pierre and D. J. Trudnowski, "Use of ARMA block processing for estimating stationary low-frequency electromechanical modes of power systems", IEEE Trans Power Syst., vol. 18, no. 1 , (2003), pp. 167-173.

[5] D. J. Trudnowski, "Estimating electromechanical mode shape from synchrophasor measurements", IEEE Trans. Power Syst., vol. 23, no. 3, (2008), pp. 1188-1195. 
[6] N. Zhou, J. W. Pierre and R. W. Wies, "Estimation of low-frequency electromechanical modes of power systems from ambient measurements using a subspace method", Proceedings of the North American Power Symposium, (2003); Rolla.

[7] J. Thambirajah, N. F. Thornhill and B. C. Pal, "A multivariate approach towards interarea oscillation damping estimation under ambient conditions via independent component analysis and random decrement”, IEEE Trans. Power Syst, vol. 26, no. 1, (2011), pp. 315-322.

[8] G. H. James, T. G. Carne and J. P. Lauffer, "The natural excitation technique for modal parameter extraction from operating wind turbines", Sandia National Laboratories, (1993).

[9] P. V. V. Reddy and L. Rajamani, "Performance Evaluation of Hypervisors in the Private Cloud based on System Information using SIGAR Framework and for System Workloads using Passmark”, International Journal of Advanced Science and Technology, vol. 70, (2014), pp. 49-60.

[10] L. Wan, M. Hong and J. Xu, "Identification of modal parameters for model of a ship hull girder under ambient excitation based on NExT/ERA method", Journal of Ship Mechanics, vol. 17, no. 7, (2002), pp. 774-784.

[11] J. N. Juang and R. S. Pappa, "An eigensystem realization algorithm for modal parameter identification and model reduction", Journal Guidance Control, vol. 8, (1985), pp. 620-627.

[12] H. Li, "Large engineering structures modal parameters identification technology", Beijing Institute of Technology Press: Beijing, (2007).

[13] Q. Wan, Y. Min and Y. Zhang, "A New Algorithm of Oscillatory Active Power Increment Distribution in Low Frequency Oscillation Study”, Automation of Electric Power Systems, vol. 32, no. 6, (2008), pp. 1-4.

[14] J. N. Juang and R. S. Pappa, "An Eigensystem Realization-Algorithm for Modal ParameterIdentification and Model-Reduction", Journal of Guidance, Control and Dynamics, vol. 8, no. 5, (1985), pp. 620-627.

[15] R. S. Pappa and K. B. Elliott, "Consistent-Mode Indicator for the Eigensystem Realization Algorithm", Journal of Guidance Control and Dynamics, vol. 16, no. 5 , (1993), pp. 852-858.

[16] C. Wu, H. Liu and J. Wang, "Parameter identification of a bridge structure based on a stabilization diagram with fuzzy clustering method", Journal of Vibration and Shock, vol. 32, no. 4, (2013), pp. 121126.

[17] P. Kundur, "Power system stability and control”, McGraw 2 Hill, (1994).

[18] G. Rogers, "Power System Oscillations", Kluwer, (2000).

\section{Authors}

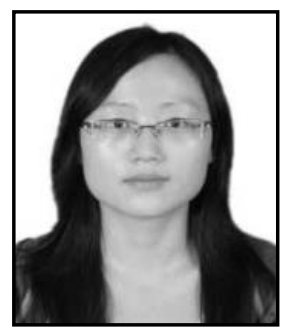

Gao Jie, She received the B.Sc.(Eng.) degree in electrical engineering from Southwest Jiaotong University School of Electrical Engineering, Chengdu, China, in 2008. He is pursuing the M.DPh.D. degree at the

Southwest Jiaotong University School of Electrical Engineering, Chengdu,China.

Currently, her main research interests are wide-area monitoring, electromechanical oscillation damping, and Signal processing applications in power system.

Wang Jia received the M.Sc.(Eng.) degree in electrical engineering from Southwest Jiaotong University School of Electrical Engineering , Chengdu,China, in 2012.

Currently, he is a engineer of Sichuan Electric Power Company-\&Measuring Center. His main research interest is the electric energy metering and electricity information acquisition.

Zhou Yang received the B.Sc.(Eng.) degree in electrical engineering from Southwest Jiaotong University School of Electrical Engineering, Chengdu, China, in 2008. He is pursuing the M.D-Ph.D. degree at the Southwest Jiaotong University School of Electrical Engineering, Chengdu,China.

Currently, his main research interests are the power quality analysis and supply variable electric technology. 
International Journal of Control and Automation Vol.9, No.1 (2016) 